\title{
Liquid metal based smart fiber sensor for human-computer interaction
}

\author{
Mingxun Chen $^{1, *}$ \\ ${ }^{1}$ Department of Electrical and Electronic Engineering, Southern University of Science and Technology Shenzhen, China
}

\begin{abstract}
Flexible electronic devices based on liquid metal fibers have attracted the attention of many laboratories in the world due to their convenient use and characteristics of being able to be woven into flexible textiles or applied directly on the body surface. In this research, we utilized the liquid metal mixed with copper particles ( $\mathrm{Cu}$-EGaIn) as the outer conductive layer of stretchable fiber, developing a highperformance composite conductive fiber based on liquid metal. The composite conductive fiber has three layers: stretchable elastic fiber core; adhesion layer; liquid metal layer. Specifically, the stretchable elastic fiber core provides the high tensile property, the adhesion layer is used to hold the liquid metal on the fiber surface, and the liquid metal layer makes the fiber have a high electrical conductivity. This kind of fiber not only has the characteristic of high electrical conductivity of metal materials, but also can always maintain high electrical conductivity even in large-scale tensile state. Therefore, we developed a tension sensor based on liquid metal intelligent fiber for human-computer interaction.
\end{abstract}

\section{INTRODUCTION}

Stretchable sensors have a wide application prospect in various fields, such as wearable devices [1], intelligent consumer electronics, human-computer interaction device [2] and intelligent robots. Therefore, a variety of stretchable sensors based on new flexible conductive materials have gradually attracted the attention of researchers. Compared to the traditional sensors made up of rigid materials, stretchable sensors have higher freedom and are capable to fit tightly on the surface of various complex structures such as the surface of human skin so that they can be used to detect the body movements of human or intelligent robots. (For instance, stretchable tension sensors based on the CNT fiber can be used to detect the human movements; Stretchable chemical sensors such as sweat sensors, can be used to measure the blood sugar levels by detecting the proportion of glucose in sweat without injuring the skin.) The stretchable sensor based on fibers has the advantages of simple structure, easy preparation, being woven into a variety of structures and easy adhesion on the body surface, which makes it has made great progress in recent years. At present, many kinds of flexible conductive materials are used to make stretchable conductive fibers, such as metal nanofibers [3], carbon-based nanomaterials [4], conductive hydrogels, ionic liquids [5], and organic conductive polymers. Although these flexible conductive materials have good stretchability, but their conductive properties are far lower than those of traditional solid metal materials. The development of a new material with both high stretchability and conductive properties has become an important research direction in the manufacture of a new generation of stretchable conductive fiber sensors.

Liquid metal is a kind of low melting point alloy material that can keep in liquid state at the normal temperature environment. This material not only has high conductivity and good thermal conductivity like traditional metal materials, but also possess the characteristics of liquid, which makes it extremely flexible and can withstand large-scale tensile deformation. Due to its advantages of low melting point and high conductivity, liquid metal materials have unique advantages in the preparation of flexible sensors [6], and are widely used in the manufacturing of flexible robot and wearable electronic devices [7-8]. For instance, liquid metal can be used in electronics tattoo that have an easy access to monitor physiological signals of human [9]. In addition, liquid metal has good fluidity and electrical conductivity, so researchers begin to fabricate completely flexible liquid metal electrodes that is able to improve the therapeutic effect by encapsulating them with flexible materials [10-11]. In recent years, fibrous flexible electronic devices based on liquid metal have been reported to have the advantages of simple manufacture and high tensile property, showing great application potential and gradually arousing the interest of researchers at home and abroad.

At present, researchers are using liquid metal conductive fibers in a variety of applications, such as stretchable wire [12], strain sensors and super capacitors which are made of liquid metal fibers. One of commonly fabrication of liquid metal conductive fiber is injecting gallium and indium alloy into the silicone [13], which has

*chenmingxun990405@163.com 
a high tensile properties and the ability to encapsulate the liquid metal. However, the diameter of liquid metal conductive fiber made by this method is limited by the diameter of silica gel hose, so it is difficult to make ultrafine conductive fiber [14]. Besides, 3D printing can also be utilized to directly prepare high-precision liquid metal conductive fibers [15]. But fibers manufactured in this way have poor tensile properties and are not suitable for mass production.

In this paper, aiming at the flaws existing in the manufacturing process of liquid metal conductive fiber, we put forward a new preparation method of liquid metal conductive fibers. Specifically, this method adopts nested multi-layers structure, using the polyurethane elastic fiber that has a super stretchability as carrier. Then, its surface is covered with liquid metal conductive coating and this kind of conductive fiber showed high stretchability in the tests. In the experiments, it demonstrates good stretchability, electrical stability and the ability to be used as a tension sensor to detect the movement of human body limbs, which confirms that this semi-liquid metal conductive fiber may have great application prospect in the field of human-computer interaction and intelligent robot.

\section{Material ANd Methods}

\subsection{Materials}

Liquid metal applied in the experiment is Gallium-indium eutectic alloy which is composed of $74.5 \%$ gallium and $24.5 \%$ indium, having a melting point of $15.5^{\circ} \mathrm{C}$. It was sold by Anhui Minor New Materials Co. Ltd. Additionally, the polymethacrylates (PMA) solution purchased from Hong Kong Yihui Co. Ltd was diluted by $25 \mathrm{wt} \%$ deionized water to improve its liquidity after received. Here, copper microparticles (mean diameter of $12 \mu \mathrm{m}$ ) were purchased from Beijing DK Nano Technology Co. Ltd and the polyurethane (PU) fibers which have a mean diameter of $126 \mu \mathrm{m}$ were commercially purchased.

\subsection{Synthesis of Cu-EGaln}

According to the previous research, particle internalization can lead to $\mathrm{Cu}$ particles wetted into EGaln, preparing desired $\mathrm{Cu}-\mathrm{EGaIn}$. In the process of synthesis, the EGaln $(100 \mathrm{~g})$ was prepared by placing gallium $(74.5 \%)$ and indium $(24.5 \%)$ in a beaker with a heating process $\left(200{ }^{\circ} \mathrm{C}\right)$ for one hour. In order to make sure they were fully mixed, a glass bar was used to stir the solution during the whole process. Then, the copper microparticles consisting of $15 \mathrm{wt} \%$ of EGaln were scattered on the surface of EGaln, which is followed by the immersion in the $\mathrm{NaOH}$ aqueous solution $(100 \mathrm{~mL}, 1.0 \mathrm{~mol} \mathrm{~L}-1)$. Finally, the semiliquid metal $\mathrm{Cu}$-EGaIn was formed with a constant agitation ensuring totally mixing of copper microparticles and EGaln.

\subsection{Preparation of Cu-Ga-In/Fiber}

In order to form a stable PMA coating adhered on the fiber surface, PU fibers were firstly immersed in the PMA solution for 5 seconds, which was followed by a drying process in air for 5 minutes. Then, $\mathrm{Cu}$-EGaln was spread uniformly on the fiber surface by using a brush because of the significant adhesion between PMA coating and $\mathrm{Cu}$ Ga-In. Next, two ends of PU fibers with a coating of $\mathrm{Cu}-$ EGaln were connected to copper wires. Finally, PU fibers experienced an immersion in the Ecoflex with a temperature of $60{ }^{\circ} \mathrm{C}$ for 3 minutes to complete the encapsulation of $\mathrm{Cu}$-EGaln coating.

\subsection{Characterization}

Here, the morphology of PU fiber surface and Cu-EGaln coating were observed by a scanning electron microscopy (SEM, S-4300). A camera (Canon EOS 800D) was used to record the Optical images, and a digital source meter (Keithley, Model 2400) was used to test the resistance variation of $\mathrm{Cu}-\mathrm{Ga}-\mathrm{In} /$ Fibers during stretching process.

\section{RESULTS AND DisCUSSION}

In this research, PU fibers with high stretchability were utilized to prepare conductive fibers. PU fiber is a kind of common polymer fiber material which is inexpensive and easy to manufacture. Figure $1 \mathrm{~A}$ demonstrates the fabrication process of the intelligent conductive fiber based on liquid metal (Cu-EGaIn/fiber). More specifically, this $\mathrm{Cu}-\mathrm{EGaIn} /$ fiber has four layers: 1) PU fiber located at the core 2) PMA coating on the surface of the PU fiber 3) $\mathrm{Cu}$-EGaln coating adhered to the PMA coating surface 4) The outermost Ecoflex coating layer which is used to encapsulate internal $\mathrm{Cu}$-EGaIn coating. More details of the fabrication process of $\mathrm{Cu}-\mathrm{EGaIn} /$ fiber are described in materials and methods section.

Previous research has showed that the adhesion of $\mathrm{Cu}-$ EGaIn to the substrate depends on the roughness of the microstructure of the substrate surface and the chemical interaction of liquid metal with the substrate surface. In this research, $\mathrm{Cu}$-EGaIn used is susceptible to oxidation in air, forming a layer of metallic oxide on the surface. The metallic oxide layer can form hydrogen bond interactions with PMA coating on PU fiber surface so that $\mathrm{Cu}$-EGaIn can easily form a strong adhesion to the fiber surface, as shown in the Figure 1B. Additionally, Cu-EGaIn does not tend to form a strong adhesion to the PU fibers because of the rough surface of the PU fibers. However, the existence of PMA coating on the PU fiber surface provides a smooth interface, greatly increasing the contact area between PMA coating and Cu-EGaIn coating and improving the adhesion of $\mathrm{Cu}$-EGaIn. 


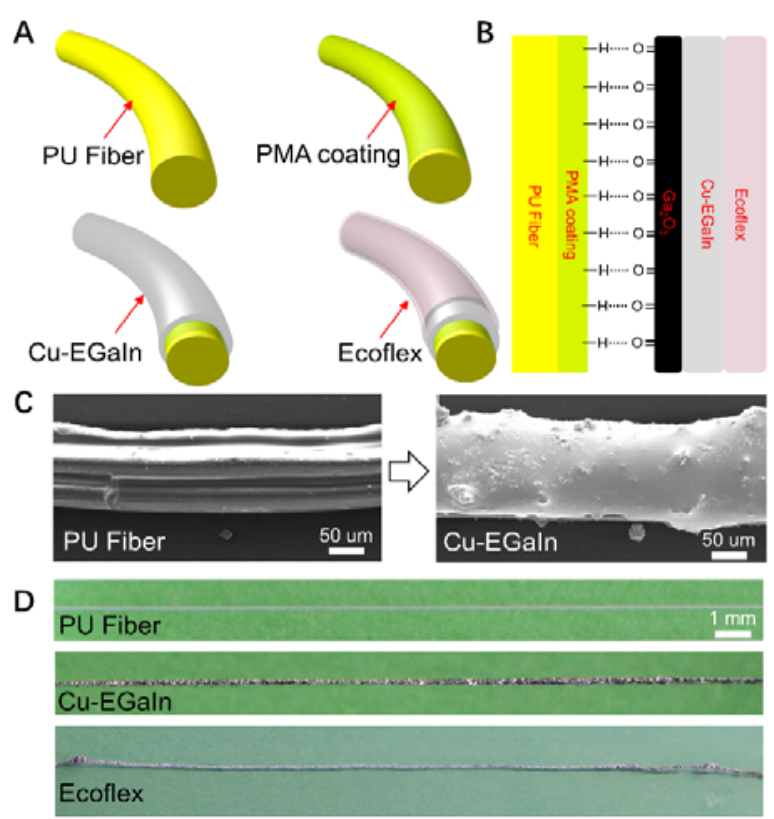

Figure 1 Preparation method of liquid metal intelligent fiber (A) Schematic diagram of the fiber preparation process (B) Adhesion principle of PMA coating to liquid metal conductive layer (C) Electron microscope images of PU fiber and liquid metal intelligent fiber (D) Physical image of liquid metal intelligent fiber

$\mathrm{Cu}$-EGaIn used in this research performs better than other common liquid metal materials in conductivity (1.94 $\times 10-7 \Omega \cdot \mathrm{m})$ and shaping ability because of the doping of $\mathrm{Cu}$ microparticles. Specifically, in the fabrication process of $\mathrm{Cu}$-EGaIn, $\mathrm{Cu}$ microparticles were uniformly mixed in liquid metal and their surfaces were infiltrated to form a liquid-bridge effect which greatly reduced the fluidity of the composite. Consequently, Cu-EGaIn coating adhered to the fiber surface is stable enough to prevent it from breaking away from the fiber surface under gravity.

According to the Figure 1C, scanning electron microscopy (SEM) images of PU fibers and Cu-EGaIn coating are demonstrated. It can be observed that $\mathrm{Cu}-$ EGaIn is uniformly covered on the fiber surface and $\mathrm{Cu}$ microparticles are scattered in the liquid metal. These microscopic images confirm the fact that the smooth interface provided by the PMA coating and hydrogen bond interactions between layers lead to a relatively strong adhesion of Cu-EGaIn to the fiber surface. Importantly, liquid metal has a higher percentage of the $\mathrm{Cu}$-EGaIn coating, maintaining considerable liquidity. Therefore, $\mathrm{Cu}$-EGaIn coating is able to deform when PU fibers are stretched under external force. Physical figures of different layers of $\mathrm{Cu}-\mathrm{EGaIn} /$ fiber are shown in the Figure 1D.

It can be seen from Figure $1 \mathrm{C}$ that the thickness of $\mathrm{Cu}-$ EGaIn coating on PU fiber surface is approximately 26-35 um. Therefore, according to the Equation (1), the theoretical resistance value of $\mathrm{Cu}-\mathrm{EGaIn} /$ fiber and the theoretical range of resistance change during streching process can be calculated. here, $\rho$ is the resistivity of $\mathrm{Cu}-$ EGaIn $(1.94 \times 10-7 \Omega \cdot \mathrm{m}), \mathrm{L}$ is the length of $\mathrm{Cu}-\mathrm{Ga}-$ $\mathrm{In} /$ fibers $(10 \mathrm{~cm}) . \mathrm{D}$ is the diameter of $\mathrm{Cu}-\mathrm{Ga}-\mathrm{In} /$ fibers, $\mathrm{d}$ is the diameter of inner fibers and $\mathrm{R}$ is the resistance of $\mathrm{Cu}$-EGaIn/fibers.

$$
R=\frac{4 \rho \cdot L}{\pi \cdot\left(D^{2}-d^{2}\right)}
$$

Figure 2 demonstrates the electrical performance of $\mathrm{Cu}-\mathrm{EGaIn} /$ fibers during stretching process. The strainresistance curve is shown in Figure 2A. Specifically, the $\mathrm{Cu}-\mathrm{EGaIn} /$ fiber (initially length of $10 \mathrm{~cm}$ ) with maximum strain of $300 \%$ shows good stretchable property. In addition, the resistance of $\mathrm{Cu}-\mathrm{EGaIn} /$ fibers increases greatly with the increase in tensile strength (from initially $4.22 \Omega$ to $41.14 \Omega$ under $300 \%$ strain). According to the calculation based on the Equation 1, the Theoretical initial resistance $\mathrm{Cu}$-EGaIn/fibers should be $3.11 \Omega$ which is slightly lower than that of practical measured value. This situation may be caused by nonuniform $\mathrm{Cu}$-EGaIn coating on the fiber surface since it can lead to a higher local resistance.

In order to show the practical application of $\mathrm{Cu}$ EGaIn/fiber as a stretchable wire, we connected a LED with $\mathrm{Cu}-\mathrm{EGaIn} /$ fiber and supply it with a $3.3 \mathrm{~V} \mathrm{DC}$ voltage. When $\mathrm{Cu}-\mathrm{EGaIn} /$ fiber is stretched to different lengths (100\% and 200\% strain), the lightness of LED did not change significantly, which indicates that $\mathrm{Cu}$-EGaIn /fiber can maintain a considerable conductivity during the stretching process to ensure a normal operation of stretchable circuit. This feature makes it possible for it to be applied to wearable devices which highly requires a stability under strain. On the other hand, Cu-EGaIn /fiber is repeatedly stretched to different lengths and its resistance is recorded in real time during this process, so that we can evaluate the electrical stability, as shown in the Figure $2 \mathrm{C}$. It can be seen from the results that $\mathrm{Cu}$ EGaIn/fiber can maintain constant and continuous resistance changes under $100 \%, 200 \%$ and $300 \%$ strain.
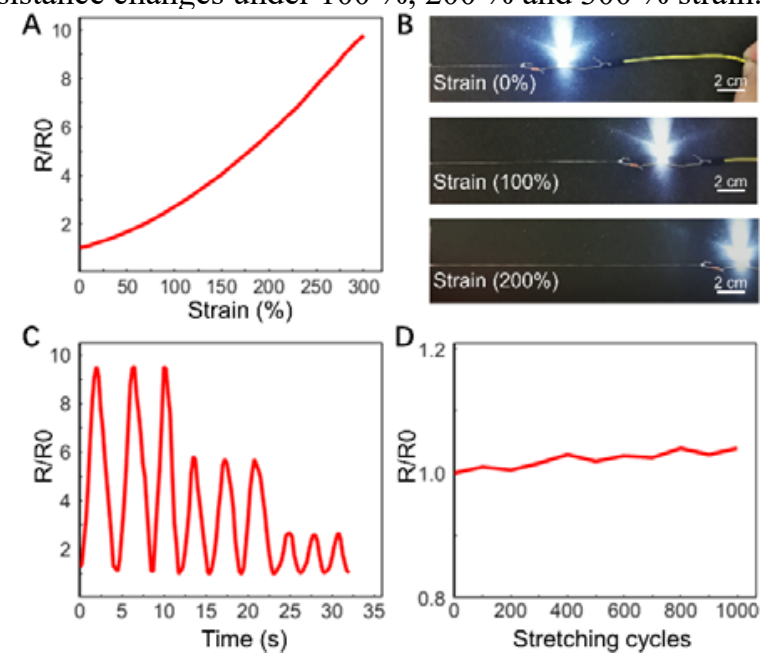

Figure 2 Electrical performance test of liquid metal intelligent fiber (A) Tension-resistance curve of liquid metal intelligent fiber (B) Feasibility of connecting liquid metal intelligent fiber to LED lamp (C) The resistance curve of liquid metal intelligent fiber under different tensile modes (D) Electrical stability test of liquid metal intelligent fiber

Finally, in order to measure the stability of $\mathrm{Cu}$ $\mathrm{EGaIn/fiber}$ after it subjected to repeated stretching, $\mathrm{Cu}$ EGaIn /fiber was stretched to $100 \%$ strain for 1000 times 
and the resistance value after every 100 times was recorded, as shown in Figure 2D. According to the results, during the process of continuously stretching, there is no significant change in resistance value of $\mathrm{Cu}$-EGaIn/fiber but there is slightly increase in its resistance. This phenomenon may be caused by the local oxidation of $\mathrm{Cu}-$ EGaIn coating which reduces the conductivity as the stretch time increases. However, this slight increase in $\mathrm{Cu}$-EGaIn /fiber is negligible compared to the high resistance values of the electronic components, proving that $\mathrm{Cu}$-EGaIn/Fiber can provide excellent stability and is suitable for long periods of working.

$\mathrm{Cu}-\mathrm{EGaIn} /$ fibers show high stretchability and high stability and only need a relatively simple fabrication process. The results mean that they can be applied as stretchable conductive fibers and fiber tension sensors, which can be applied in the fields of motion detection and human-computer interaction. In this article, we designed a tension sensor based on $\mathrm{Cu}$-EGaIn/fibers. Specifically, we applied it to the human finger model to detect the bending angle of the finger, as shown in Figure 3A. Here, Cu-EGaIn/Fibers were encapsulated in the Ecoflex film, and as the Ecoflex film stretched, the $\mathrm{Cu}-$ EGaIn/Fibers could be stretched with it. Copper wire was connected at both ends of Cu-EGaIn/Fibers and then connected to the subsequent detection circuit. Detection circuit was actually a resistance-voltage conversion circuit that was used to convert the resistance signals of thermistor into voltage signals. Then, the microprocessor MSP430G2553 was used to collect and digitize the voltage signals in real time.
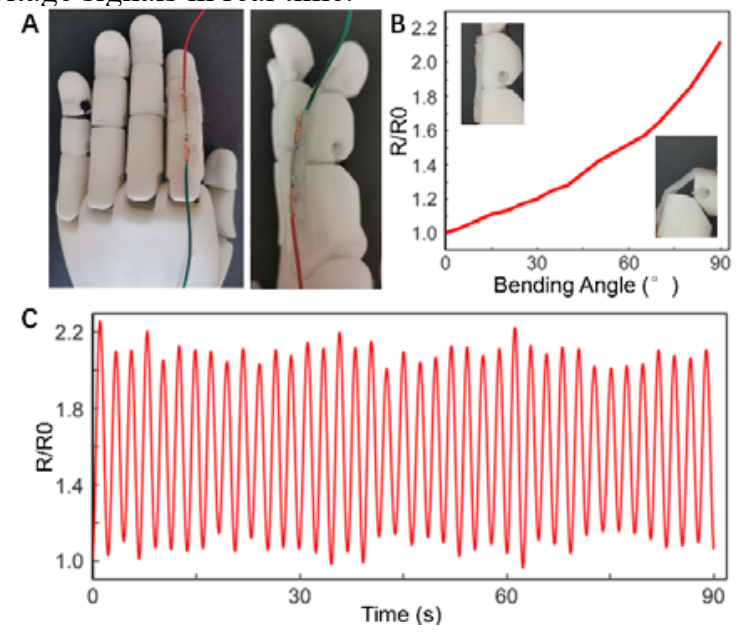

Figure 3 Liquid metal intelligent fiber for human-computer interaction (A) Liquid metal intelligent fiber for tension sensors B) Joint bending Angle-resistance curve (C)

Electrical stability test of liquid metal tension sensor

According to the Figure 3B, the fingers of the model can be bent to about $90^{\circ}$. During this process, tension sensor attached to the knuckles was stretched accordingly, and the $\mathrm{Cu}-\mathrm{EGaIn} /$ Fiber was then stretched with it, causing its resistance to increase. Curve in the Figure 3B shows the relationship between the resistance of tension sensor and the bending angles. More specifically, relative resistance of tension sensor at $90^{\circ}$ is 2.12 times as large as that of original state, which means that the resistance variation is clear enough for us to calibrate. Thus, in practical application, bending angles of fingers can be calculated by measuring the resistance of tension sensor according to the obtained curve.

In addition, we made multiple bends in the model knuckle and recorded resistance changes of the tension sensor during this process in real time, as shown in the Figure 3C. It can be seen from the results that the resistance of this tension sensor based on $\mathrm{Cu}$ EGaIn/Fibers can steadily increase with the finger bending angles. When the knuckle is not bent, the resistance of the sensor will return to its original state. According to the data in the experiment, the maximum change of relative resistance is $1.27(\mathrm{R} / \mathrm{R} 0$ is from 0.96 to 2.23) and the minimum change of relative resistance is $0.87(\mathrm{R} / \mathrm{R} 0$ is from 1.14 to 2.01$)$, which do not require a complex system to judge the state of fingers because of the considerable changing range of relative resistance. Therefore, this tension sensor can be utilized as a wearable human-computer interaction system to detect and collect the movement information of user's hand for personal health detection, sports entertainment and other applications.

\section{CONCLUSION}

In conclusion, this research demonstrated a new kind of intelligent composite fibers based on Cu-EGaIn. Compared to traditional stretchable liquid metal based conductive fibers fabricated by perfusion method, the $\mathrm{Cu}-$ EGaIn material was served as the conductive coating of composite fibers, which was located in the outermost layer. In addition, the purpose of exposing semi-liquid metal coating in the outermost layer was to make full use of the low melting point and contact wetting of $\mathrm{Cu}$-EGaIn materials, realizing that the composite fiber was capable to remain conductive with the core fiber in the stretching state. Based on the previous research about liquid metal intelligent fiber done in the lab, we improved the fabrication process of intelligent composite fibers to solve the problem that the gallium indium alloy with good fluidity was difficult to adhere to the fiber surface stably. More specifically, this semi-liquid material doped with solid metal particle was capable to maintain an original adhesion state stably in a period of time as well as retain the low melting point and contact wetting of liquid metal. Furthermore, in order to further improve the adhesion of semi-liquid metal materials on the surface of these fibers, we attached a layer of PMA coating that is highly adherent to $\mathrm{Cu}$-EGaIn materials to the surface of the core fibers.

This conductive fiber can be widely used as flexible and elastic conductor. Besides, due to its excellent and stable working characteristics, it can also be used as a fiber-type tension sensor. Specifically, based on the braided property of the intelligent fiber, liquid metal fiber enabled tension sensor developed in this research shows its application prospect in the field of human-computer interaction. However, it is noted that there are still some shortcomings of this technology. For example, the diameter of the core fiber of the fiber can be further reduced to fabricate smaller fiber sensors; most of the intelligent fibers are manufactured by hand and cannot 
meet the requirements of mass production; the electrical properties of the intelligent fibers vary because of artificial fabrication under different situations. In future research, it can be expected that this liquid metal intelligent composite fiber can be widely used in fields such as human-computer interaction, wearable electronics and intelligent robots.

\section{REFERENCES}

1. Suji Choi, Jinkyung Park, Wonji Hyun, Jangwon Kim, Jaemin Kim, Young Bum Lee, Changyeong Song, Hye Jin Hwang, Ji Hoon Kim, Taeghwan Hyeon, Dae-Hyeong Kim, "Stretchable Heater Using Ligand-Exchanged Silver Nanowire Nanocomposite for Wearable Articular Thermotherapy ", ACS Nano, Washington, vol. 9, pp. 6626-6633, May 2015.

2. Khan Yasser, Ostfeld AminyE, Lochner Claire M, Pierre Adrien, Arias Ana C, "Monitoring of Vital Signs with Flexible and Wearable Medical Devices ", Adv. Mater., Weinheim, vol. 28, pp. 4373-4395, February 2016.

3. Huang Wei-Ran, He Zhen, Wang Jin-Long, Liu Jian-Wei, Yu Shu-Hong, "Mass Production of Nanowire-Nylon Flexible Transparent Smart Windows for PM2.5 Capture ", iScience, Amsterdam, vol. 12, pp. 333-341, February 2019.

4. Lou Zheng, Chen, Shuai, Wang Lili, Jiang Kai, Shen Guozhen, "An ultra-sensitive and rapid response speed graphene pressure sensors for electronic skin and health monitoring ", Nano Energy, Amsterdam, vol. 23, pp. 7-14, March 2016.

5. Kim Chong Chan, Le Hyun Hee, Oh Kyu Hwan, Sun Jeong Yun, "Highly stretchable, transparent ionic touch panel ", Science, Washington, vol. 353, pp. 682-687, August 2016.

6. Biao Ma, Chengtao Xu, Junjie Chi, Jian Chen, Chao Zhao, and Hong Liu, "A Versatile Approach for Direct Patterning of Liquid Metal Using Magnetic Field ", Adv. Funct. Mater., Weinheim, vol. 29, pp. 1901370.1-1901370.8, March 2019.

7. Long Teng, Shichao Ye, Stephan Handschuh-Wang, Xiaohu Zhou, Tiansheng Gan, "Liquid Metal-Based Transient Circuits for Flexible and Recyclable Electronics ", Adv. Funct. Mater., Weinheim, vol. 29, pp. 1808739, January 2019.
8. Qian Wang, Yang Yu, Jun Yang, Jing Liu, "Fast Fabrication of Flexible Functional Circuits Based on Liquid Metal Dual-Trans Printing ", Adv. Mater., Weinheim, vol. 27, pp. 7109-7106, September 2015.

9. Guo Rui, Sun Xuyang, Yao Siyuan, Duan Minghui, Wang Hongzhang, Liu Jing, Deng Zhongshan, "Semi-Liquid-Metal-(Ni-EGaIn)-Based Ultraconformable Electronic Tattoo ", Adv. Mater. Technol., New Jersey, vol. 4, pp. 1990183, May 2019.

10. Jeong Yu Ra, Kim Jeonghyun, Xie Zhaoqian, Xue Yeguang, Won Sang Min, Lee Geumbee, Jin Sang Woo, Hong Soo Yeong, Feng Xue, Huang Yonggang, Rogers John A., Ha Jeong Sook, "A skin-attachable, stretchable integrated system based on liquid GaInSn for wireless human motion monitoring with multi-site sensing capabilities ", NPG Asia Mater., Berlin, vol. 9, pp. e443, October 2017.

11. Tavakoli M., Malakooti M. H., Paisana H., Ohm Y., Marques D. G., Alhais Lopes P., Piedade A. P., de Almeida A. T., Majidi C., "EGaIn-Assisted RoomTemperature Sintering of Silver Nanoparticles for Stretchable, Inkjet-Printed, Thin-Film Electronics ", Adv. Mater., Weinheim, vol. 30, pp. e1801852, May 2018.

12. Guo R., Wang H., Sun X., Yao S., Chang H., Wan H., Liu J., Zhang Y., "Semiliquid Metal Enabled Highly Conductive Wearable Electronics for Smart Fabrics ", ACS Appl. Mater. Inter., Washington, vol. 11, pp. 30019-30027, November 2019.

13. Lu-Yu Zhou, Qing Gao, Jun-Fu Zhan, Chao-Qi Xie, Jian-Zhong $\mathrm{Fu}$, Yong $\mathrm{He}$, "Three-Dimensional Printed Wearable Sensors with Liquid Metals for Detecting the Pose of Snakelike Soft Robots ", ACS Appl. Mater. Inter., Washington, vol. 10, pp. 23208-23217, June 2018.

14. Shu Zhu, Ju-Hee So, Robin Mays, Sharvil Desai, William R. Barnes, Behnam Pourdeyhimi, Michael D. Dickey, "Ultrastretchable Fibers with Metallic Conductivity Using a Liquid Metal Alloy Core", Adv. Funct. Mater., Weinheim, vol. 23, pp. 23082314, December 2013.

15. Boley J. William, White Edward L., Chiu George T. C., Kramer Rebecca K., "Direct Writing of Gallium-Indium Alloy for Stretchable Electronics ", Adv. Funct. Mater., Weinheim, vol. 24, pp. 35013507, February 2014. 